\title{
WHY AND HOW TO CAPTURE THE SEMANTICS OF WEB USER INTERFACES
}

\author{
Claudia Steinberger and Joachim Frießer \\ Department of Applied Informatics, Universität Klagenfurt \\ Universitätsstraße 65-67, 9020 Klagenfurt, Austria
}

\begin{abstract}
Structured data on the Web has become very important over the last few years. Schema.org has proven to be the most recognized vocabulary for structuring data on the Web. However, most annotations using Schema.org's vocabulary describe exclusively content on websites. Today, these structured content data are mainly consumed by search engines to automatically browse websites with high speed and accuracy and to take over search efforts for humans. Annotated web user interfaces can hardly be found on websites or applications. Schema.org's offered vocabulary to describe actions in a structured way plays a minor role on websites or applications and is mainly applied on rich emails. Also most structured data tools and platforms do not support the annotation of actions triggered via the user interface of a website or application. In this paper we motivate the importance of a semantic description of web user interfaces and present possible application areas to consume these structured data. Thereon, we investigate the suitability of Schema.org for the semantic enrichment of web user interfaces. Since there are hundreds of classes and properties in Schema.org, we present a conceptual model of Schema.org classes and properties suitable to annotate user interfaces of websites or applications. We also show to what extent interactive elements on HTML5 websites or applications can be automatically mapped to these model elements. As a proof of concept we introduce Schemator, our WYSIWYG tool to support the semantic enrichment process of web user interfaces. Schemator is not only a comfortable structured data tool but acts also as a SAAS platform to store and manage structured web interface data separated from the underlying websites and applications.
\end{abstract}

\section{KEYWORDS}

Semantic Web, Web User Interface, Schema.org, Structured Data

\section{INTRODUCTION}

Today the challenge in designing websites lies on structured, high quality data. During the last decade amazing progress has been made in enriching data in the Web with semantic data that provides a machine-readable "meaning" of information and is layered over the information that is provided for people.

To structure data on the web, many vocabularies have been developed (Vandenbussche 2017) but Schema.org has proven to be the most widely acknowledged one (Mika 2015) (Guha et al. 2016). The big search engine providers have been the main promoters of Schema.org and semantic data enrichment of data in the Web. They developed a vocabulary ${ }^{1}$ which is compact and easy to use to describe "things" on the Web and to make them interoperable and machine read- and understandable. Today structured data is consumed by search engine bots to correctly classify and interpret website content. It is used to feed their knowledge graphs (Paulheim 2017) and to provide better search results on end user requests. Better positions and representations in organic search results, knowledge cards or widespread online map services in turn motivate website designers and operators to provide structured data on their websites. Today over 10 million websites use Schema.org to markup some of their content. Compared to websites with a rather static set of pages that offer viewers with content details, interactive web applications, where user engagement is higher and dynamic content and actions predominate, structured data is behind.

This paper describes why and how to semantically enrich user interfaces of websites or applications beyond content and individual actionable elements. What would a machine-readable 'meaning' of website or application user interface elements and thus the understanding of their features enable? Structured user

${ }^{1}$ https://schema.org/docs/schemas.html 
interface data could be used e.g. for the conversational handling of websites or applications using intelligent personal assistants or chatbots, for workflow management or to search the Web for special features. Structured user interface data could also be used by intelligent assistance systems as a means to guide a user through an application (Steinberger, Michael 2019).

In this paper we investigate the suitability of Schema.org to semantically enrich websites or application user interface elements. As there exist hundreds of classes and properties in Schema.org we present a conceptual model of appropriate Schema.org classes and properties to annotate interaction elements. We also show how interactive elements on HTML5 websites or applications can be automatically scanned and mapped to this model elements. As a proof of concept we introduce Schemator, our WYSIWYG tool to support the semantic enrichment process of web user interfaces. Schemator is not only a comfortable structured data tool but acts also as a SAAS platform to store and manage structured web interface data separated from the underlying websites and applications.

This paper is structured as follows: Chapter 2 presents application scenarios of structured data in the Web today focusing on the Schema.org vocabulary. Chapter 3 deals with the elements and relations of a web user interface, its semantics we intent to make machine-readable and how to describe them using Schema.org. We present a conceptual model of the necessary Schema.org excerpt and present an approach to map interactive HTML-constructs on websites to target classes of this model. Chapter 4 introduces the Schemator, a tool we built to evaluate our approach. Schemator supports the semantic user interface enrichment process as a SaaS and offers a WYSIWYG interface loading the web site or application under annotation. Chapter 5 summarizes our findings and gives an outlook to identified further research challenges.

\section{STRUCTURED DATA ON THE WEB - PRODUCERS AND CONSUMERS}

In recent years, amazing advances have been made in enriching data on the Web with semantic data that provide a machine-readable "meaning" of information. The number one consumer of structured data on the Web are machines, not humans. A human user never sees structured text on a web page, but e.g. web-bots or other "intelligent agents" do. Therefore, semantic interoperability and the exchange of data with an unambiguous meaning plays a major role in the Semantic Web.

Schema.org has proven to be the most acknowledged vocabulary to structure data on the Web. Schema.org is a collaborative community project promoted by the big search engine providers with the mission to create and maintain a vocabulary for structured data used on webpages, in email messages, and beyond. Schema.org can be encoded in RDFa, Microdata or JSON-LD and interpreted and individually used by any tool or service that receives the structured data (Mika 2015).

Today, structured data are mainly consumed by search engines to automatically browse websites with high speed and accuracy and to take over search efforts for humans. The annotation with structured data increases the chance of a website to appear as a rich result, for example a rich snippet, a featured snippet or quick answer. Rich results are in turn also often used as the sources for answers in voice search. As a result, Schema.org structured data are mainly produced and provided on websites in the context of semantic search engine optimization (Henry 2018) for content types with mostly direct or indirect commercial relevance like products, places, events, books, videos, films, persons, organizations and more.

Although most uses of the Schema.org vocabulary explain "things", there exist elements on websites and applications that are not content, but ways to take action. Schema.org also offers a vocabulary to describe actions in a structured way, simply called Actions. But structured action data can hardly be found on websites or applications. As Schema.org has grown, it offers various mechanisms for community extension as a way of adding more detailed descriptive vocabulary that builds on the Schema.org core. Nevertheless, the most extensions deal with content (Hepp 2015) (Barker et al. 2016) too, although some try to use and extend the vocabulary e.g. to annotate HTTP APIs to enable the automation of service calls (Simsek et al. 2018).

Annotating data for web content is complicated and costly but website designers and operators have been motivated in return for better search engine ranking and results. Producing structured data web designers have to deal with the vocabulary to use, how to simply create the desired annotations and how to represent, to store and to publish them. The demand for tools to produce structured data has grown. There exist a lot of annotation tools and plugins with different levels of automation. Most of them are not widely used because they are embedded in a CMS that enforces access rights to the CMS backend and usually only support certain 
Schema.org classes. Some tools are strictly decoupled from the underlying website and generate and store annotations separated from the content. But the complexity of the offered vocabulary combined with missing WYSIWYG functionality often overburden the average producer of structured data. To counteract this (Khalili, Auer 2013) (Kärle et al. 2017) have worked on solutions for the creation, publication and distribution of semantic annotations of content in an easier and more intuitive way.

Besides search engines, knowledge graphs and knowledge vaults are one of the major consumers of structured data. Knowledge vaults are typically large scale knowledge bases that collect and express their data using a shared semantic vocabulary. Some are open for all to use and can be queried using an API. Their main goal is to store millions of entities and reliable, associated facts about those entities. Some of the more prominent players in development include Google Knowledge Graph and Knowledge Vault, Bing's Satori, DBPedia or Wikidata (Paulheim 2017) (Färber et al. 2018). Google uses its knowledge graph e.g. to generate knowledge cards shown in the search console.

Also other intelligent agents started to consume Schema.org annotations. The Schema.org markup "Speakable"2 for instance enables language assistants to reproduce the contents of a website linguistically on demand. Annotations of content and actions in emails can help them to stand out from the rest in the inbox, be consumed to enable the user to call go-to actions very easily (e.g. order an item, check in a flight, reset password) or to summarize and to highlight key information in the inbox app. Also intelligent personal assistants start to support voice-activated actions based on annotated emails.

To the best of our knowledge, there is no approach to semantically enrich the user interface of a website or application. The machine-readable 'meaning' of all relevant website or application interactive elements and thus the understanding of its user interface functionality enables new possibilities for intelligent agents. Let's think, for example, on conversational handlings of websites and applications using intelligent personal assistants or chatbots, web application workflow management agents, online help agents, the interoperability of web applications with intelligent assistance systems (Steinberger, Michael 2018) or the possibility to search web sites and applications for certain functions.

With our approach we want to semi automatically generate, validate, store and publish semantic annotations beyond content and individual go-to action elements with the primary focus on the user interface of websites or applications. Our approach aims to create an open knowledge base that can be read and edited in the crowd, separating structured user interface data from the underlying websites and applications.

In the following we investigate how to semantically describe a web user interface with Schema.org and how to scan and map HTML5 interaction elements to our conceptual model of selected Schema.org classes. With Schemator we present a web application whose main purpose is to make the annotations of website or application functionality easy and intuitive. But Schemator is not only a platform for generation, editing and storage of annotations but also to validate and publish them. By this way Schemator offers a free and open knowledge base of structured user interface data collected in the crowd, separating structured user interface data from the websites they describe.

\section{SEMANTIC DESCRIPTION OF WEB USER INTERFACES}

Before we take a closer look at the Schemator platform, we present our research process on the semantic description of web user interfaces with structured data (see figure 1): First in (I), we selected typical website or application use cases like the homepage of our University, a well-known online shop and an online banking application. Based on these use cases, we identified and categorized those user interactions that we wanted to consume later in conversations with intelligent personal assistants, chatbots or intelligent assistance systems (Michael et al. 2018)(Steinberger, Michael 2018).

\footnotetext{
${ }^{2}$ https://schema.org/speakable
} 


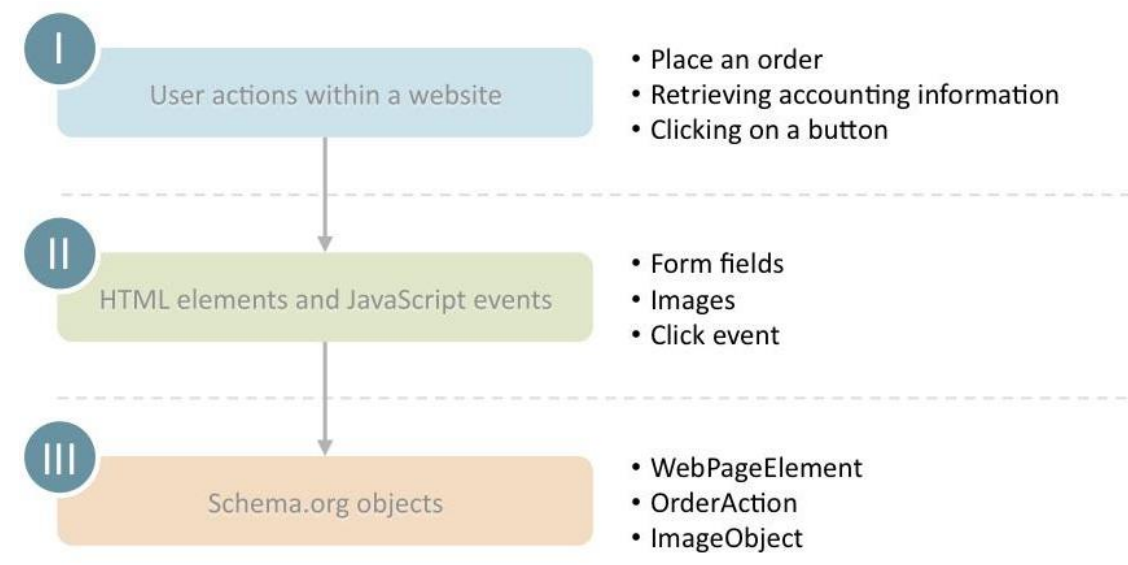

Figure 1. Step-by-step process for creation of the semantic user interface data following (Frießer 2019)

Thus, not every clickable HTML element on a website or application has been relevant for us. Fortunately, some of the big companies like Apple, Google, Mozilla and Microsoft have been working on the development of the HTML living standard (WHATWG). A concept named "inertness" was introduced there: If an element is identified as inert, the user agent (e.g. the browser) hast to ignore this element for user interaction (e.g. text search or selection). So we could identify a number of interactive HTML elements focusing only those covering at least an activation behavior as specified in (WHATWG): "change", "click", "contextmenu", "dblclick", "mouseup", "pointerup", "reset", "submit" and "touchend" (Frießer 2019).

The next challenge was to semantically describe the interaction elements found with the vocabulary of Schema.org. Schema.org covers a large catalog of classes and properties, but only a small part of the language is necessary to describe the identified interaction elements. Based on our use cases we developed in (III) a conceptual model of the Schema.org classes for that purpose. Figure 2 shows our target structured data vocabulary for the web user interface description:

A website or application can be described by using the class WebApplication. Every single page of a web application is characterized by the class WebPage that obtains respectively persist of multiple instances of the class WebPageElement. They are all subtypes of CreativeWork and therefore inherit properties such as name or description. MediaObjects and the more specific types AudioObject, VideoObject and ImageObject can be linked by applying the property associatedMedia. Every associated WebPageElement has a potentialAction property that is a result to another WebPage or Action. Finally a certain way for navigating through the different web pages may described as a BreadCrumbList.

What triggers the interaction with a WebPageElement can be described using the property hasPotentialAction referencing an Action. Each Action has a name, a description and a result. Latter is of type CreativeObject (and thus e.g. again a WebPage). Several specific Action types like AchieveAction, SearchAction, CreateAction and more are predefined in Schema.org (Steinberger, Michael 2019).

Our goal was to create structured user interface data according to this Schema.org excerpt model. Figure 3 shows a simplified example of the JSON-LD description of the launch site of Amazon.com (seen with Google SD-Tool $)^{3}$. To create this kind of structured data, we wanted to map HTML user interface elements as automatically as possible to the selected Schema.org classes and properties. Thus, in (II) we investigated the HTML elements and Javascript events on our use case websites or applications in more detail. What semantic information about an identified user interface element can be retrieved from the document object model (DOM)?

\footnotetext{
${ }^{3}$ https://search.google.com/structured-data/testing-tool
} 


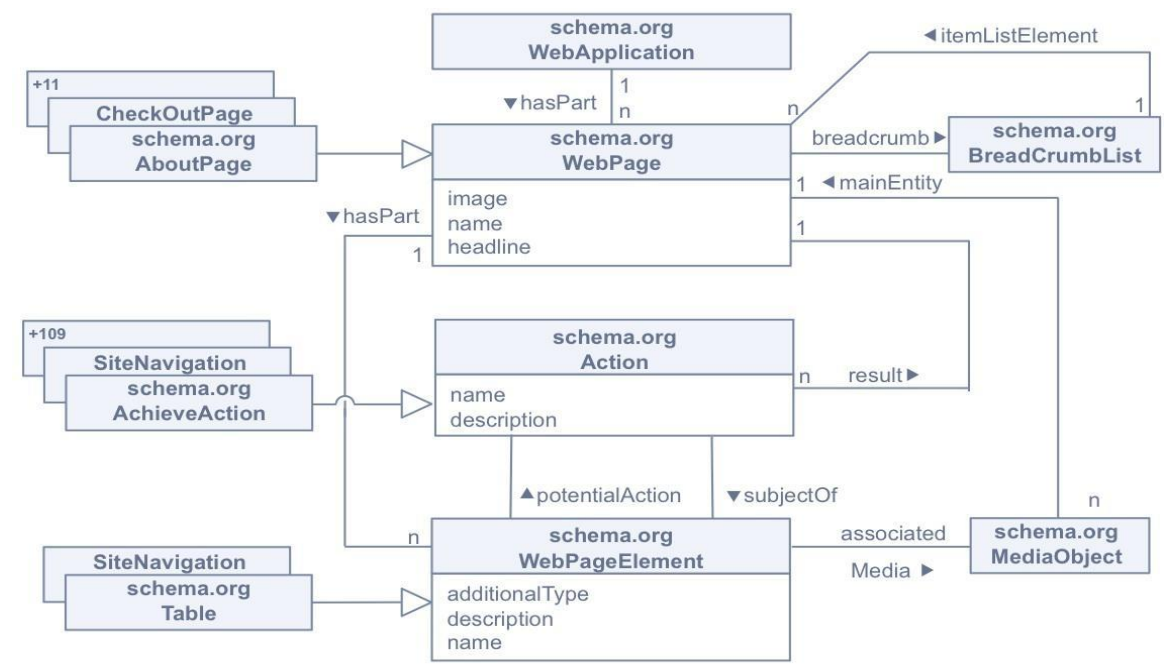

Figure 2. Conceptual model of Schema.org excerpt necessary for user interface description (Frießer 2019)
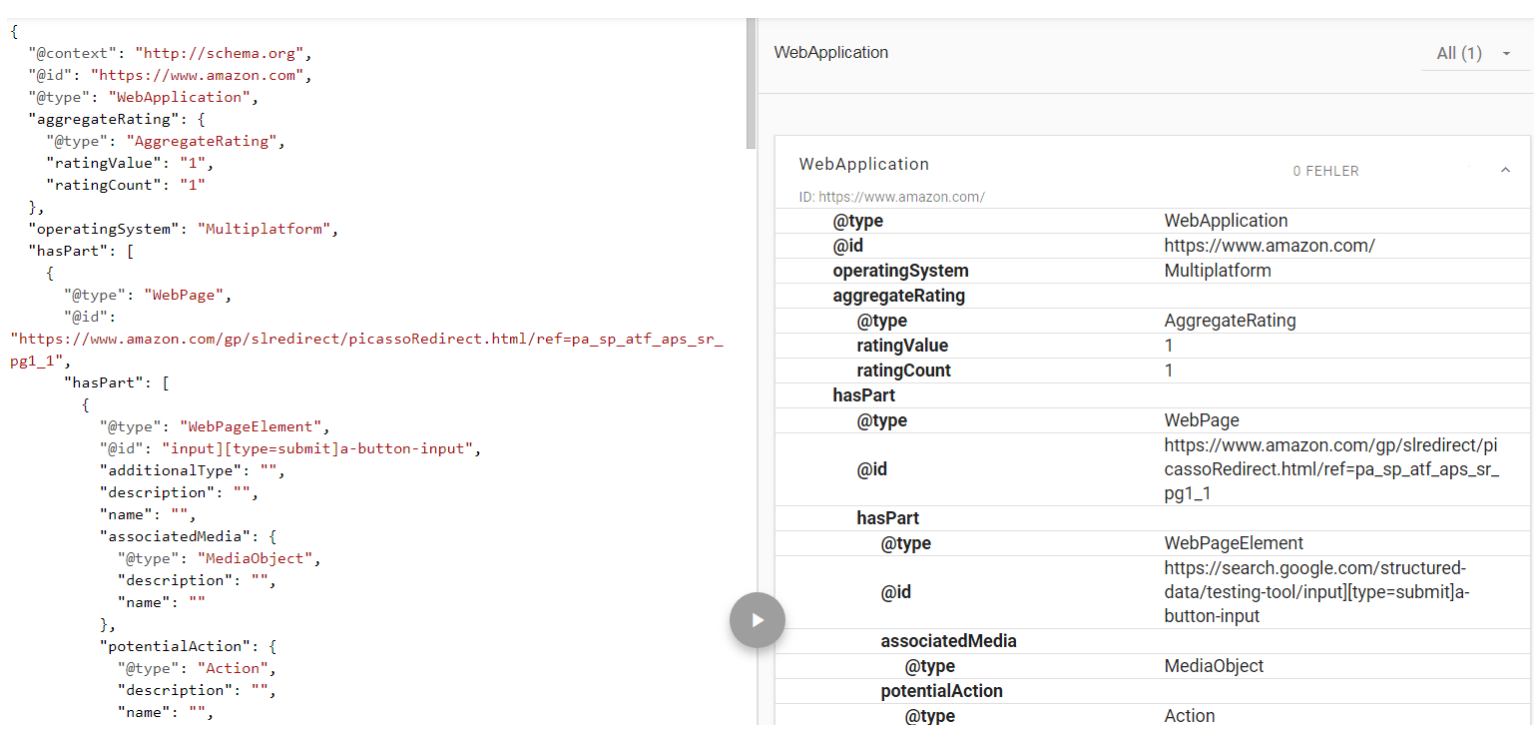

Figure 3. Structured user interface data of the launch site of Amazon.com seen with Google SD-Tool

Our investigations showed that the required semantic user interaction data could not be reliably and completely extracted from the identified HTML elements on the websites or applications (Frießer 2019). Some naming conventions have been specified in the semantics section of (WHATWG), which state that the name of a HTML tags can have a certain meaning and a clue for the purpose, e.g. the "addr" tag is used for an address. But such conventions are not reliably adhered to. Even the label or rather a text can be a hint for the functionality of e.g. button, but if an icon is used, a machine won't be able to guess or "see" what the purpose of the control is.

However, the knowledge gained about which interactive controls are associated with an event is sufficient for an initial draft of a website's structured user interface data, which must then be manually checked and supplemented. The next chapter takes a closer look onto the tool support for a semi-automatic semantic description of web user interfaces and defines the user roles and the architecture of our Schemator platform. 


\section{SCHEMATOR: STRUCTURED USER INTERFACE DATA MANAGEMENT PLATFORM}

Schemator is a WYSIWYG tool to support the semantic enrichment process of web user interfaces. Schemator is not only a comfortable semi-automatic structured data tool but acts also as a SAAS platform to store and manage structured web interface data separated from the underlying websites and applications. Figure 4 shows the roles that users can assume working with Schemator: (1) Tagger, (2) Content Admin, (3) Data Consumer and (4) Model Designer.

The (1) Tagger's main task is to register and to annotate the user interface of websites or applications with Schemator and to produce structured user interface data in a semi-automatic this way: With his knowledge of the functionality of a particular website or application and the way a user typically performs certain tasks there, the Tagger creates structured data semi-automatically in a WYSIWYG mode and stores it on the platform. After a desired website has been selected or registered, he can view the website in "browser mode" superimposed into Schemator in a WYSIWYG representation. To edit existing or add new semantics to the depicted website or application, the Tagger can switch from "browser mode" to "annotate mode" (see figure 5). Interactive elements and their properties are displayed now in the element catalog on the left side of the user interface. By clicking on an element on the left, Schemator highlights the control (e.g. an input field or a button) and scrolls it into the Tagger's view if necessary. Now the Tagger is able to focus the interactive element and to edit the corresponding structured data. An advantage of using Schemator is that the Tagger does not need to have any previous knowledge of Schema.org. The input fields are dynamically generated based on the Schema.org excerpt model, as shown in figure 2.

To prevent villains from abusing the platform, the (2) Content Admin validates annotations, grants or denies user rights or bans unwanted websites. The (3) Data Consumer consumes structured data for further purposes in form of JSON-LD documents. In fact, the structured data can be also embedded as an external link target of a script tag. Finally the role of the (4) Model Designer is to define/edit the model of the Schema.org vocabulary that is supported by Schemator (at the moment the excerpt in figure 2 is supported). That means, Schemator is flexibly customizable this way.

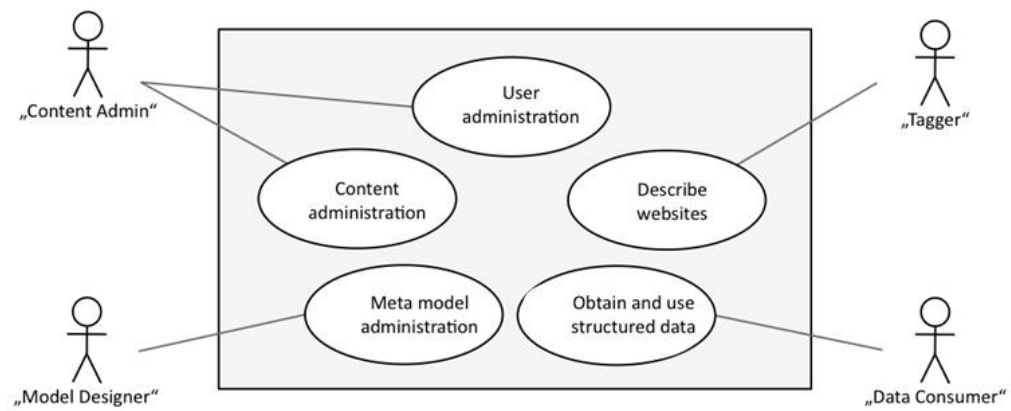

Figure 4. Use case diagram of the Schemator platform

The prototype of Schemator was implemented as a web application in the context of a master thesis at the Universität Klagenfurt and covers the main functionality for the "Tagger" and the "Data Consumer" roles (Frießer 2019). Schemator consists of a client and a server component. As figure 6 shows, due to security reasons the user agent does not directly interact with a website or application under consideration. The server component additionally acts like an "in between web proxy". 


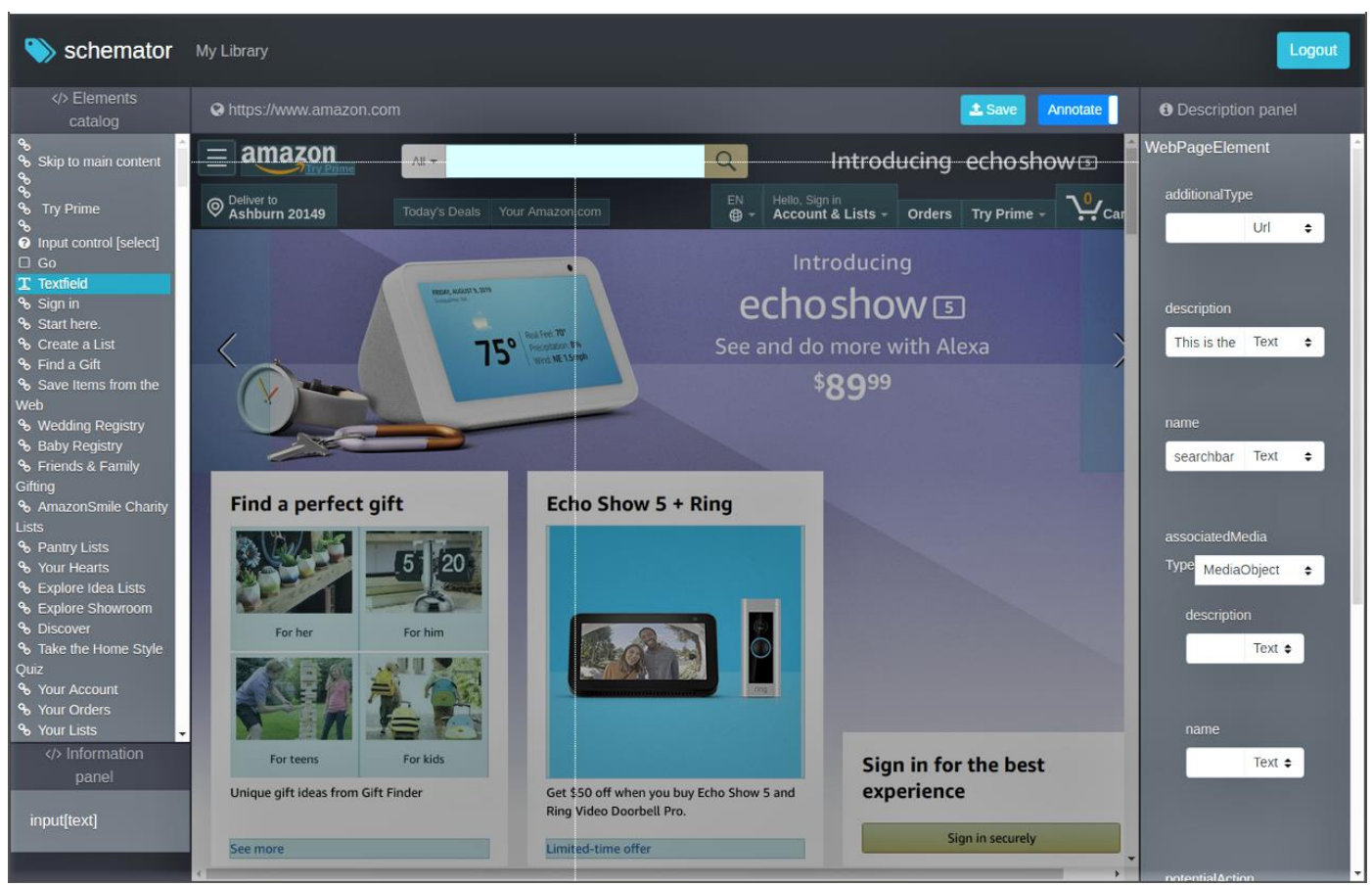

Figure 5. User interface of Schemator showing Amazon.com in the "annotate" mode

The Content Checker scans the website or application and only if it considered as safe, than Schemator acts like a real webserver proxy and saves the downloaded files in the Web Cache Storage. Schemator's main data representation and interchange format is JSON-LD, even for the representation of the Schema.org excerpt model as presented in chapter three. Because of the strong use of JSON-LD, MongoDB ${ }^{4}$, a document-driven database, is used for persistent data management in the server component. The task of the client component of the schema is to recognize the potential "tag-worthy" interactive elements of the website.

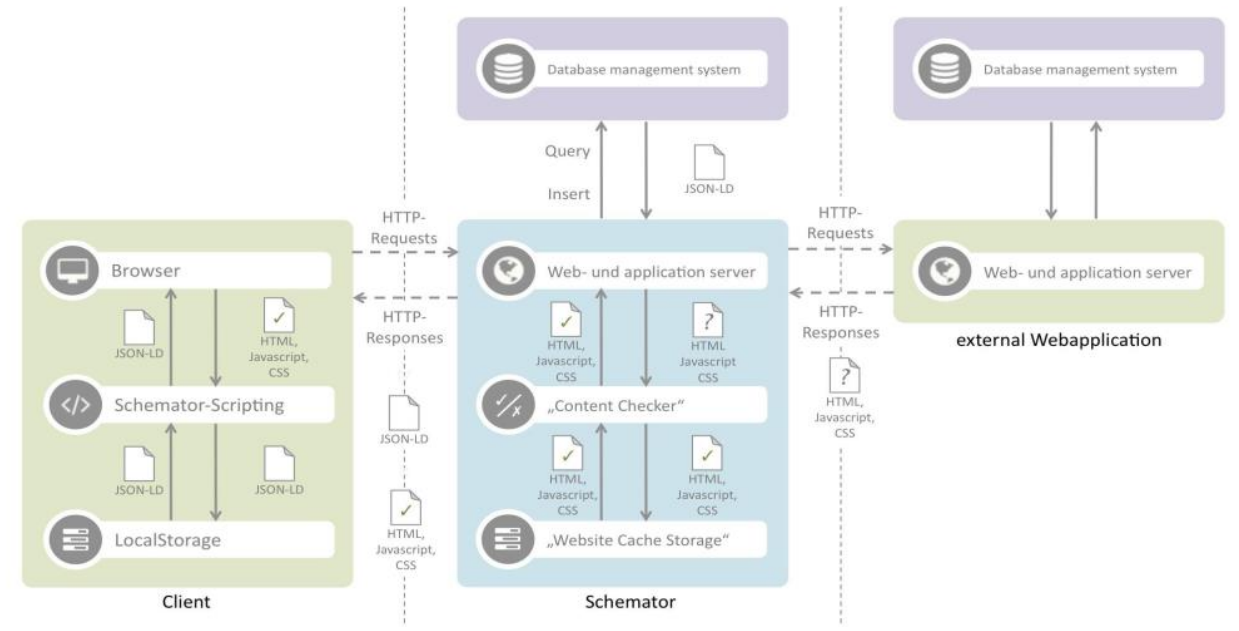

Figure 6. System architecture of the Schemator following (Frießer 19)

Some common frameworks like Bootstrap and jQuery ${ }^{5}$ are used for the user interface and business logic, the latter framework is mainly responsible for the interactive control detection. Finally the LocalStorage as defined in (WHATWG) is used to temporary save valuable information of already annotated items. This architecture allows a disaster recovery of the data if the browser crashes during the tagging process.

\footnotetext{
${ }^{4}$ https://www.mongodb.com/

${ }^{5}$ https://getbootstrap.com/; https://jquery.com/
} 


\section{CONCLUSION AND OUTLOOK}

In this paper we motivated the importance of structured user interface data and showed that the Schema.org vocabulary is suited also to semantically enrich the user interface of websites or applications. We presented a conceptual model of appropriate Schema.org concepts to annotate interaction elements and showed possibilities and limits to map interactive HTML5 elements to this Schema.org model elements.

As a proof of concept we presented Schemator, our WYSIWYG tool to support the semantic enrichment process of web user interfaces. The existing version of the Schemator does not yet fulfill all use cases shown in figure 4, but it covers the core functionality. The tagging process works and the resulting JSON-LD can be consumed by intelligent agents. Schemator is not only a comfortable structured data tool but also serves as a SAAS platform to store and manage structured web interface data separated from the underlying websites and applications.

In future we will continue developing the next version, collect structured user interface data of selected websites and applications and evaluate the Schemator. We will also work on the integration of Schemator into our active assisted living projects (Steinberger, Michael 2019).

\section{REFERENCES}

Barker, P., \& Campbell, L. M. (2016). Technology Strategies for Open Educational Resource Dissemination. In P. Blessinger, \& B. T. J., Open Education: International Perspectives in Higher Education (pp. 51-70). doi:10.11647/OBP.0103.03.

Färber, M., Bartscherer, F., Menne, C., \& Rettinger, A. (2018). Linked data quality of dbpedia, freebase, opencyc, wikidata, and yago. Semantic Web, 9(1), 77-129.

Frießer, J. (2019). Semantische Aufbereitung von Benutzeroberflächen von Webapplikationen für die aktive Assistenz, mit $H M B S$, diploma thesis, to be published in July2019.

Guha, R. V., Brickley, D., \& Macbeth, S. (2016). Schema. org: evolution of structured data on the web. Communications of the ACM, 59(2), 44-51.

Hepp, M. (2015, June). The web of data for e-commerce: Schema. org and GoodRelations for researchers and practitioners. In International Conference on Web Engineering (pp. 723-727). Springer, Cham.

Kärle, E., Şimşek, U., \& Fensel, D. (2017). semantify. it, a Platform for Creation, Publication and Distribution of Semantic Annotations. arXiv preprint arXiv:1706.10067.

Khalili, A., \& Auer, S. (2013, October). Wysiwym authoring of structured content based on schema.org. In International Conference on Web Information Systems Engineering (pp. 425-438). Springer, Berlin, Heidelberg.

Michael, J., Steinberger, C., Shekhovtsov, V. A., Al Machot, F., Ranasinghe, S., \& Morak, G. (2018). The HBMS story. Enterp. Model. Inf. Syst. Arch, 13, 345-370.

Mika, P. (2015). On Schema.org and why it matters for the web. IEEE Internet Computing, 19(4), 52-55.

Paulheim, H. (2017). Knowledge graph refinement: A survey of approaches and evaluation methods. Semantic web, 8(3), 489-508.

Şimşek, U., Kärle, E., \& Fensel, D. (2018). Machine readable web apis with schema.org action annotations. Procedia Computer Science, 137, 255-261.

Steinberger, C., \& Michael, J. (2018). Towards cognitive assisted living 3.0. In 2018 IEEE International Conference on Pervasive Computing and Communications Workshops (PerCom Workshops) (pp. 687-692). IEEE.

Steinberger, C.,Michael J. (2019). Using Semantic Markup to Boost Context Awareness for Assistive Systems in Feng Chen, Rebaca I. García-Betances, María Fernanda Cabrera-Umpiérrez, Liming Chen, Chris Nugent: Smart Assisted Living, (to be published in October 2019).

Vandenbussche, P. Y., Atemezing, G. A., Poveda-Villalón, M., \& Vatant, B. (2017). Linked Open Vocabularies (LOV): a gateway to reusable semantic vocabularies on the Web. Semantic Web, 8(3), 437-452.

WHATWG. https://whatwg.org/, retrieved on 2019/05/24 